\title{
NEUTRON ACTIVATION COOL-DOWN OF THE TOKAMAK FUSION TEST REACTOR
}

\author{
H. W. Kugel, ${ }^{\mathrm{a}}$ G. Ascione, ${ }^{\mathrm{a}}$ C. Tilson Jr., ${ }^{\mathrm{a}}$ and A. Kumar ${ }^{\mathrm{b}}$ \\ ${ }^{a}$ Princeton Plasma Physics Laboratory Princeton University \\ P. O. Box 451, Princeton NJ 08543 \\ ${ }^{\mathrm{b}}$ School of Engineering and Applied Science, \\ University of California, Los Angeles, CA 90095-159710
}

\begin{abstract}
TFTR final operations and post-shutdown neutron activation measurements were made. Ionization chambers were used to follow TFTR activation during operations and after shutdown. Gamma-ray energy spectroscopy measurements were performed to characterize TFTR activation at accessible vessel-bays, and on sample hardware removed from structures at various distances from the vessel. The results demonstrate long-lived activations from common, commercially available materials used in the fabrication and field engineering of TFTR. The measurements allow characterization of residual TFTR neutron activation, the projection of residual activation decay, and benchmarking of low activation issues simulations.
\end{abstract}

\section{INTRODUCTION}

The Tokamak Fusion Test Reactor (TFTR) began high power deuterium-tritium (D-T) fueled operations in December 1993, completed operations in April 1997, and is presently in a shutdown, safe-keeping mode. ${ }^{1}$ The achieved performance includes central ion temperatures up to $44 \mathrm{keV}$, electron temperatures up to $13 \mathrm{keV}$, fusion power yields up to $10.7 \mathrm{MW}$, and a D-T fusion energy yield of $1.55 \mathrm{GJ}$, corresponding to a total D-T neutron yield of more than $5.52 \times 10^{20}$. There was also about 0.15 GJ of D-D fusion energy yield. These operations involved about 21,670 high power discharges, including about 1000 D-T discharges of which about 750 D-T discharges involved at least one neutral beam injecting tritium, and about 250 discharges into which tritium gas was puffed into the tokamak with all neutral beams injecting deuterium. This work involved the safe handling and processing about $100 \mathrm{~g}$ of tritium. This resulted in manageable long-term neutron activation of the vessel and surrounding hardware. In this paper, we report the results of post-shutdown measurements made for the purpose of characterizing residual TFTR neutron activation, projecting residual activation decay, exploring low activation issues, and investigating options for future radioactive waste management.

\section{EXPERIMENTAL CONDITIONS}

Extensive simulations of TFTR radioactivation have been performed previously using estimates of the Test Cell material density distribution. ${ }^{2,3}$ The materials used in the construction of the TFTR facility are known but due to the evolution of the experimental requirements the facility grew in complexity, and hence, the exact material density distribution has been difficult to quantify. However, during D-T operations, the configuration remained relatively unchanged to meeting maintenance, reliability, and performance requirements. Fig. 1 shows a partial schematic plan view of the TFTR concrete Test Cell showing the relative locations of the vessel, neutral beam injection systems, the vacuum system pump ducts, and the activation measurements. The characteristics of the Test Cell shielding and radiation field have been described previously. $^{4}$

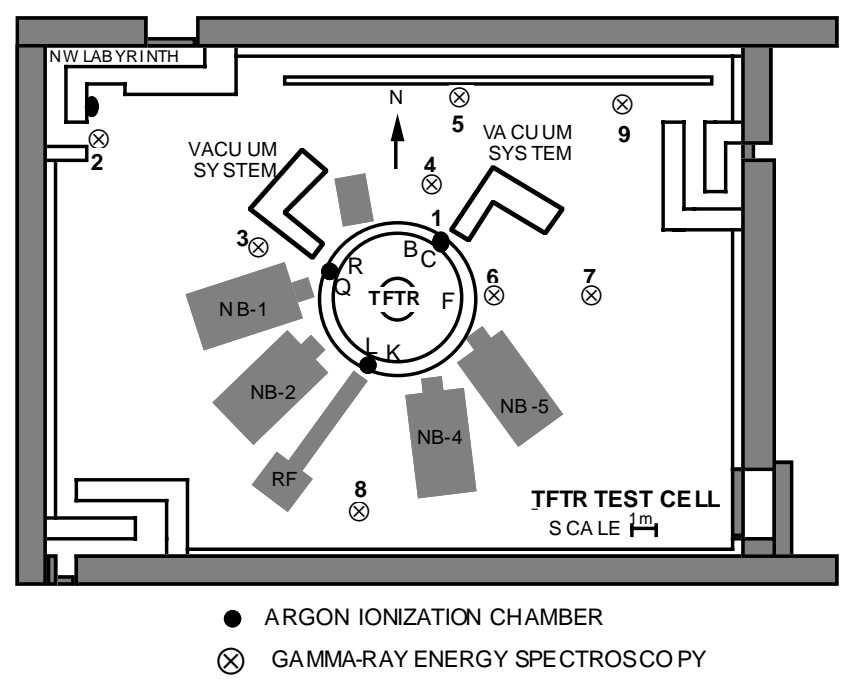

Fig. 1 Partial schematic plan view of the TFTR Test Cell showing the location of the activation measurements relative to the vessel, and other components. 


\section{ACTIVATION MEASUREMENTS}

\section{A. Ionization Chamber Measurements}

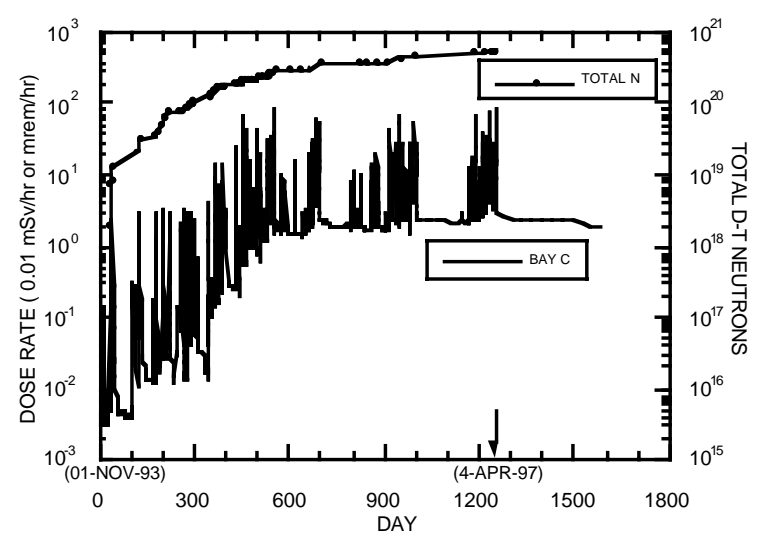

Fig. 2. The dose rate at Vessel Bay-C, $1.2 \mathrm{~m}$ from lower vessel (Fig.1, Location 1) as measured at 6:30 am each morning with Ionization Chamber.

Activation measurements were performed with four gamma-ray Argon Ionization Chamber detectors. Three detectors were located near the vessel $(1.2 \mathrm{~m}$ outboard of vessel at $\mathrm{R}=4.9 \mathrm{~m}$, and $2 \mathrm{~m}$ below mid-plane) and the fourth at a distance at $\mathrm{R}=24.5 \mathrm{~m}$ at about $1 \mathrm{~m}$ below midplane. These measurements were performed automatically every 15 minutes and computer archived. During experimental operations, TFTR operated in the pulse mode with pulse lengths 5 to $10 \mathrm{sec}$ with a dwell time between pulses about 8 to 15 minutes, from about 0800 hrs to 2300 hrs, 5 days per week. Fig. 2 shows Ionization Chamber measurements of the TFTR activation gamma dose rate at Bay $\mathrm{C}$, about $1.2 \mathrm{~m}$ from the lower vessel (Fig.1, Location 1), as measured at $0630 \mathrm{hrs}$ each morning, about $7.5 \mathrm{hrs}$ after the end of operations on the previous day. The activity shown in Fig. 2 exhibits a large increase after each day of D-T operation which then rapidly decayed to a long-term level higher than prior to the sequence of D$\mathrm{T}$ discharges. A similar behavior was exhibited in the activations measured at the other TFTR locations but with differences attributed to different local materials and shielding. During D-T operations, all of the ionization chamber locations exhibited a burst of activation during D$\mathrm{T}$ operations which decayed to an activation level higher than before the D-T burst (about 1-10 $\mathrm{mSv} / \mathrm{hr}$ after $7 \mathrm{hr}$ ). Typical contact dose rates at a vessel flange 2 weeks after D-T operations were about $1 \mathrm{mSv} / \mathrm{hr}$. The midplane contact dose rates about 400 days after shutdown were about 0.3 $\mathrm{mSv} / \mathrm{hr}(30 \mathrm{mR} / \mathrm{hr})$. The measured dose rates exhibited complex decay rates due to many contributing short-lived (hours to days), intermediate (months), and long-lived (years) activities. ${ }^{2}$ The vessel activation decayed with a effective fast $3.2 \mathrm{hr}$ decay half-life, followed by about an 18 $\mathrm{hr}$ decay half-life, and finally a longer decay half-lives in the range from $230 \mathrm{~d}$ and longer. At shorter times, the observed decay half-lives indicate the importance of ${ }^{56} \mathrm{Mn}$ $(2.6 \mathrm{hr})$ from the ${ }^{56} \mathrm{Fe}(\mathrm{n}, \mathrm{p}){ }^{56} \mathrm{Mn}$ reaction, and ${ }^{24} \mathrm{Na}(15 \mathrm{hr})$ from the ${ }^{27} \mathrm{Al}(\mathrm{n}, \alpha)^{24} \mathrm{Na}$ reaction due to iron and aluminum structural materials, and other short-lived high intensity activations due to unforeseen impurities in certain construction materials. The longer lived activity results from $\mathrm{Fe}-\mathrm{Cr}-\mathrm{Ni}$ related radioisotopes, such as ${ }^{54} \mathrm{Mn}$, and ${ }^{60} \mathrm{Co}$ generated by various reaction channels and the ${ }^{58} \mathrm{Co}$ from activation of structural alloys containing $\mathrm{Ni}$ such as, SS-304, SS-316, Nitronic, and Inconels. ${ }^{2}$ The long-lived contributions became increasingly observable as the shorter-lived activities decayed and thereby improved the signal-to-background ratio for sensitive gamma-ray energy spectroscopy measurements.

\section{B. Gamma-ray Energy Spectroscopy Measurements}

Gamma-ray energy spectroscopy measurements described here were performed at selected Test Cell locations about 326 days after shutdown, to identify the long-term isotopic contributions to the observed decay activities. The measurements were performed with a high purity $\mathrm{Ge}$, high efficiency ( $41 \%$ efficiency relative to $\mathrm{NaI}$ at $1333 \mathrm{keV}$ ), portable gamma-ray detector connected to a PCbased signal processing and data analysis system. The results exhibited considerable dependence on location due to local variations in material density and isotopic composition, as was found with the Argon Ionization Chamber measurements described above.

Fig. 3 shows the gamma-ray energy spectrum measured at the Northwest Entrance to the Test Cell, at vessel Bay-R, at major radius $\mathrm{R}=22.4 \mathrm{~m}$ (Fig.1, Location 2) for $301 \mathrm{~s}$ with a detection dead-time of $0.96 \%$. The location background dose was $0.4 \mu \mathrm{Sv} / \mathrm{hr}$ (40 $\mu \mathrm{rem} / \mathrm{hr})$. This background is significantly less than a previously reported measurement at the same location with a similar detector system performed $14 \mathrm{hrs}$ after a day of intense D-T operation when the activity was $7 \mu \mathrm{Sv} / \mathrm{hr}$ and the system dead-time was $7 \%$ using $1.1 \mathrm{~cm}$ wide $\mathrm{Pb}$ slits in front of the detector. In the case of the present measurements, the dominant activities shown in Fig. 3 are ${ }^{54} \mathrm{Mn}$ ( $\gamma$-ray energy $=834.8 \mathrm{keV}),{ }^{60} \mathrm{Co}(1173.2 \mathrm{keV}, 1332.5 \mathrm{keV})$, and ${ }^{40} \mathrm{~K}$ $(1460.8 \mathrm{keV})$. The ${ }^{54} \mathrm{Mn}$ (half life $=312 \mathrm{~d}$ ) activity is attributed to the ${ }^{55} \mathrm{Mn}(\mathrm{n}, \quad 2 \mathrm{n}){ }^{54} \mathrm{Mn}$ and ${ }^{54} \mathrm{Fe}(\mathrm{n}, \mathrm{p}){ }^{54} \mathrm{Mn}$ reactions from the activation of structural alloys (e.g., SS304,-316,-321, Nitronic-33, and Inconels-600,-718,-750). The ${ }^{60} \mathrm{Co}(5.26 \mathrm{yr})$ activity is generated through various reaction channels in structural materials. The ${ }^{40} \mathrm{~K}(1.27 \mathrm{x}$ $10^{9} \mathrm{yr}$ ) activity is attributed to natural background radiation from the concrete in the Test Cell shielding structure. An 


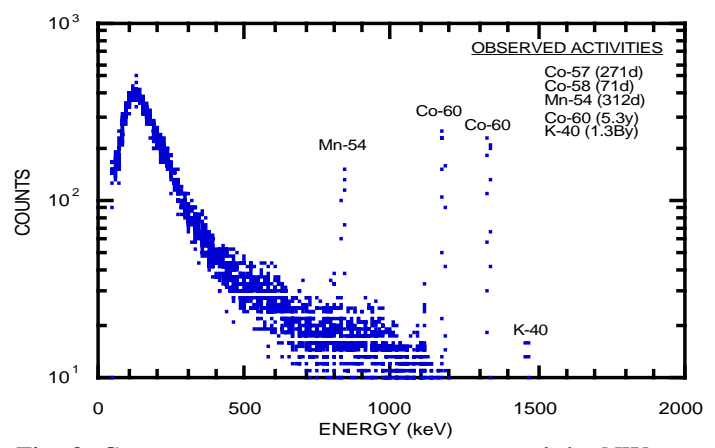

Fig. 3. Gamma-ray energy spectrum measured the NW entrance to the Test Cell at $\mathrm{R}=22.4 \mathrm{~m}$ (Fig. 1, Location 2).

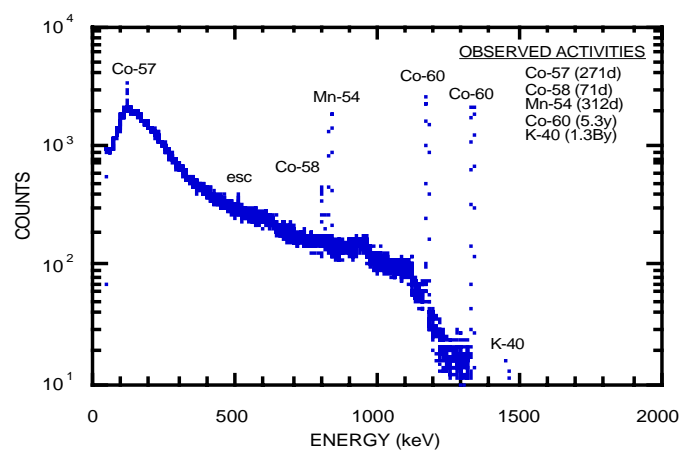

Fig. 4. Gamma-ray energy spectrum measured at Vessel Bay-R/Q at $\mathrm{R}=10.7 \mathrm{~m}$ (Fig.1, Location 3).

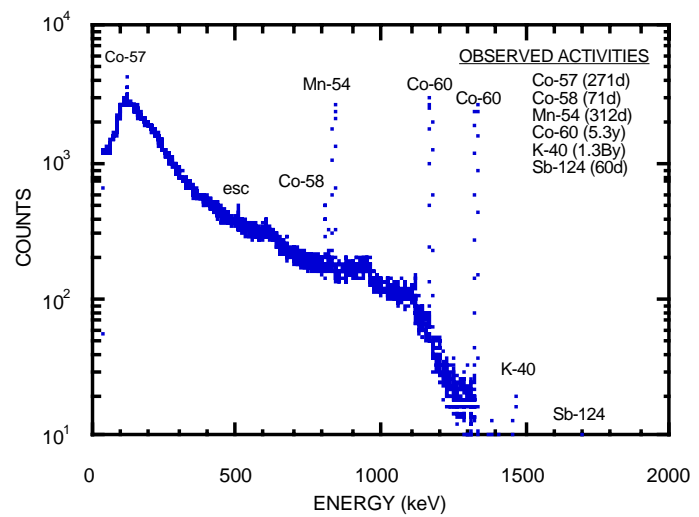

Fig. 5. Gamma-ray energy spectrum measured at Vessel Bay-B at $\mathrm{R}=9.8 \mathrm{~m}$ (Fig.1, Location 4).

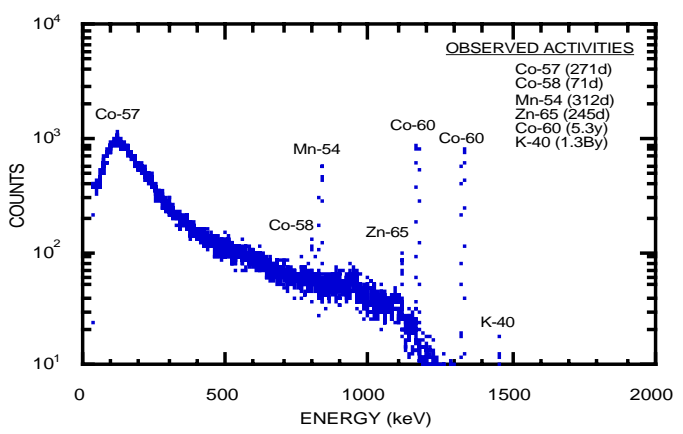

Fig. 6. Gamma-ray energy spectrum measured at Vessel Bay-K at $\mathrm{R}=15.3 \mathrm{~m}$ (Fig.1, Location 8).

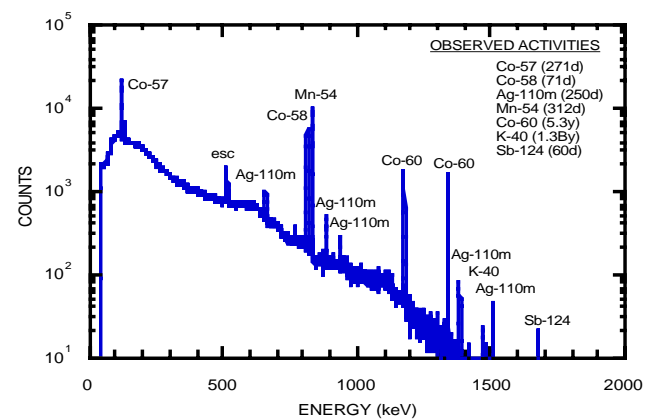

Fig. 7. Gamma-ray energy spectrum measured at the RF antenna and feedthrough assembly after removal from in-vessel service at Bay-L (Fig.1, Location 9).

indication of ${ }^{57} \mathrm{Co}(122.1 \mathrm{keV})$ and ${ }^{58} \mathrm{Co}(810.8 \mathrm{keV})$ were also observed, but were more evident at other measurement locatons (refer below).

Fig. 4 shows the gamma-ray energy spectrum measured at almost the same toroidal angle, passing between the NW Pump-Duct and a Neutral Beam Injection system, at vessel Bay-R/Q but closer to the vessel at $\mathrm{R}=10.7 \mathrm{~m}$ (Fig.1, Location 3) for $309 \mathrm{~s}$ with detection deadtime of $3.1 \%$. The location background dose rate was 2 $\mu \mathrm{Sv} / \mathrm{hr}(200 \mu \mathrm{rem} / \mathrm{hr})$. The dominant activities include those shown in Fig.3, and in addition, the ${ }^{57} \mathrm{Co}$ (122.1 $\mathrm{keV})$ and ${ }^{58} \mathrm{Co}(810.8 \mathrm{keV})$. The ${ }^{57} \mathrm{Co}(270.9 \mathrm{~d})$ activity is attributed to the ${ }^{58} \mathrm{Ni}(\mathrm{n}, \mathrm{d}){ }^{57} \mathrm{Co}$ and ${ }^{58} \mathrm{Ni}(\mathrm{n}, \quad \mathrm{n} \text { 'p })^{57} \mathrm{Co}$ reactions, and the ${ }^{58} \mathrm{Co}(70.8 \mathrm{~d})$ activity to the ${ }^{58} \mathrm{Ni}(\mathrm{n}$, p) ${ }^{58} \mathrm{Co}$ reaction from nearby nickel alloys.

Fig. 5 shows the gamma-ray energy spectrum measured at vessel Bay-B at $\mathrm{R}=9.8 \mathrm{~m}$ (Fig.1, Location 4) for $305 \mathrm{~s}$ with a detection dead-time of $4.1 \%$. This location was between the NE Pump-Duct and the Li Pellet Injection system. The background dose rate was $2.5 \mu \mathrm{Sv} / \mathrm{hr}(250$ 
$\mu \mathrm{rem} / \mathrm{hr})$. As in the case of Fig. 4, at this similarly close location, the dominant activities include those shown in Fig. 4. In addition, the spectrum exhibits a weak indication of ${ }^{124} \mathrm{Sb}(1690 \mathrm{keV})$ (not seen in the Fig.4 semi-log plot). After intense D-T operations, short-lived ${ }^{122} \mathrm{Sb}(2.75 \mathrm{~d})$ was observed, and is attributed to components of a protective tape used to wrap cable installations. The weak ${ }^{124} \mathrm{Sb}$ activity may have a similar origin, or may be contributed by components which occur in lead and brazing alloys. A similar spectrum with weaker intensity was measured at vessel Bay-B at $\mathrm{R}=15.5 \mathrm{~m}$ (Fig.1, Location 5).

Gamma-spectra energy were measured at Vessel Bay-F, at $\mathrm{R}=8.8 \mathrm{~m}$ (Fig.1, Location 6) and $\mathrm{R}=14 \mathrm{~m}$ (Fig.1, Location 7). The location background dose rates were 2 $\mu \mathrm{Sv} / \mathrm{hr}(200 \mu \mathrm{rem} / \mathrm{hr})$ and $1.2 \mu \mathrm{Sv} / \mathrm{hr}(120 \mu \mathrm{rem} / \mathrm{hr})$, respectively; the Ge gamma-ray detection dead-times were $3.6 \%$ and $1.5 \%$, respectively. This region is flanked by the NE Pump-Duct on one side, and is part of the toroidal arc from Bays-A to $-F$ containing a high concentration of diagnostic hardware of various materials. The measured gamma-ray energy spectra exhibited the same activities identified in Fig. 5 but in different intensities. The results are summarized in Table 1.
Fig. 6 shows the gamma-ray energy spectrum measured at Vessel Bay-K on the south side of TFTR at R $=15.3 \mathrm{~m}$ (Fig.1, location 8) for $303 \mathrm{~s}$ with a detection dead-time of $1.2 \%$. This location was between a Neutral Beam Injection system and an array of RF wave-guides running radially toward the vessel mid-plane. The location background dose rate was $1.1 \mu \mathrm{Sv} / \mathrm{hr}(110 \mu \mathrm{rem} / \mathrm{hr})$. The dominant activities include those exhibited at Bays-B and $\mathrm{F}$, and in addition ${ }^{65} \mathrm{Zn}(1115.4 \mathrm{keV})$. The ${ }^{65} \mathrm{Zr}(245 \mathrm{~d})$ activity may have resulted from the ${ }^{64} \mathrm{Zn}(\mathrm{n}, \gamma){ }^{65} \mathrm{Zn}$ (natural has $4.6 \%{ }^{64} \mathrm{Zn}$ ), and the ${ }^{66} \mathrm{Zn}(\mathrm{n}, 2 \mathrm{n}){ }^{65} \mathrm{Zn}$ (natural $\mathrm{Zn}$ has $27.9 \%{ }^{66} \mathrm{Zn}$ ) reactions from $\mathrm{Zn}$ containing copper alloys in the $\mathrm{RF}$ antenna wave-guide structures.

Fig. 7 shows the gamma-ray energy spectrum measured for $322 \mathrm{~s}$ with a detection dead-time of $7 \%$ at a distance of about $1 \mathrm{~m}$ at the RF antenna and feedthrough assembly that had been removed from its port location at Vessel Bay-L and placed within a tritium containment tent in the NE corner of the Test Cell at $\mathrm{R}=20 \mathrm{~m}$ (Fig.1, Location 9). The location background dose rate was 5 $\mu \mathrm{Sv} / \mathrm{hr} \quad(500 \mu \mathrm{rem} / \mathrm{hr})$. This background rate was significantly higher than comparable major radius locations in the Test Cell and is attributed predominantly to the activation of the RF antenna assembly. As was observed at locations near similar construction materials, the dominant

Table 1. Summary of the gamma-ray energy spectroscopy measurements shown in Fig.s 3-7. All measurements were made with the same detector 326 days after shutdown. Refer to text for discussion of net activity in detector counts per minute (CPM).

\begin{tabular}{|c|c|c|c|c|c|}
\hline $\begin{array}{l}\text { Location } \\
\text { (Refer to Fig.1) }\end{array}$ & $\begin{array}{l}\text { Isotope, } \gamma \text {-ray } \\
\text { energy }(\mathrm{keV})\end{array}$ & $\begin{array}{l}\text { Net Activity } \\
\text { (CPM) }\end{array}$ & $\begin{array}{l}\text { Location } \\
\text { (Refer to Fig.1) }\end{array}$ & $\begin{array}{l}\text { Isotope, } \gamma \text {-ray } \\
\text { energy }(\mathrm{keV})\end{array}$ & $\begin{array}{l}\text { Net Activity } \\
\text { (CPM) }\end{array}$ \\
\hline $\begin{array}{l}\text { NW Entrance } \\
\text { Bay-R } \\
\mathrm{R}=22.4 \mathrm{~m} \text {, } \\
(2)\end{array}$ & $\begin{array}{l}\text { Mn-54, (835) } \\
\text { Co-60, (1173) } \\
\text { Co-60, (1332) } \\
\text { K-40, }(1461)\end{array}$ & $\begin{array}{l}116 \\
258 \\
253 \\
14.4\end{array}$ & $\begin{array}{l}\text { Bay-F } \\
R=8.8 \mathrm{~m} \\
(6)\end{array}$ & $\begin{array}{l}\text { Co-57, (122) } \\
\text { Co-58, (811) } \\
\text { Mn-54, (835) } \\
\text { Co-60, (1173) } \\
\text { Co-60, (1332) } \\
\text { K-40, (1461) } \\
\text { Sb-124, (1690) }\end{array}$ & $\begin{array}{l}792 \\
228 \\
1899 \\
2582 \\
2487 \\
16.7 \\
9.0\end{array}$ \\
\hline $\begin{array}{l}\text { Bay-R/Q } \\
\mathrm{R}=10.7 \mathrm{~m} \\
(3)\end{array}$ & $\begin{array}{l}\text { Co-57, (122) } \\
\text { Co-58, (811) } \\
\text { Mn-54, (835) } \\
\text { Co-60, (1173) } \\
\text { Co-60, (1332) } \\
\text { K-40, (1461) }\end{array}$ & $\begin{array}{l}1056 \\
1451 \\
1578 \\
2638 \\
2467 \\
11.8\end{array}$ & $\begin{array}{l}\text { Bay-F } \\
R=14 \mathrm{~m} \\
(7)\end{array}$ & $\begin{array}{l}\text { Co-57, }(122) \\
\text { Co-58, (811) } \\
\text { Mn-54, (835) } \\
\text { Co-60, }(1173) \\
\text { Co-60, (1332) } \\
\text { K-40, }(1461) \\
\text { Sb-124, (1690) }\end{array}$ & $\begin{array}{l}252 \\
82 \\
632 \\
995 \\
932 \\
12.4 \\
1.2\end{array}$ \\
\hline $\begin{array}{l}\text { Bay-B } \\
R=9.8 \mathrm{~m} \\
(4)\end{array}$ & $\begin{array}{l}\text { Co-57, (122) } \\
\text { Co-58, (811) } \\
\text { Mn-54, (835) } \\
\text { Co-60, (1173) } \\
\text { Co-60, (1332) } \\
\text { K-40, (1461) } \\
\text { Sb-124, (1690) }\end{array}$ & $\begin{array}{l}1095 \\
287 \\
2308 \\
3048 \\
2867 \\
8.2 \\
11.2\end{array}$ & $\begin{array}{l}\text { Bay-K } \\
R=15.3 \mathrm{~m} \\
(8)\end{array}$ & $\begin{array}{l}\text { Co-57, }(122) \\
\text { Co-58, (811) } \\
\text { Mn-54, (835) } \\
\text { Zn-65, }(1115) \\
\text { Co-60, (1173) } \\
\text { Co-60, (1332) } \\
\text { K-40, (1461) }\end{array}$ & $\begin{array}{l}196 \\
54 \\
523 \\
76 \\
921 \\
866 \\
14.2\end{array}$ \\
\hline $\begin{array}{l}\text { Bay-B } \\
R=15.5 \mathrm{~m} \\
(5)\end{array}$ & $\begin{array}{l}\text { Co-57, (122) } \\
\text { Co-58, (811) } \\
\text { Mn-54, (835) } \\
\text { Co-60, }(1173) \\
\text { Co-60, (1332) } \\
\text { K-40, (1461) } \\
\text { Sb-124, (1690) }\end{array}$ & $\begin{array}{l}382 \\
134 \\
865 \\
989 \\
972 \\
16.5 \\
0.4\end{array}$ & $\begin{array}{l}\text { At Removed } \\
\text { RF Antenna } \\
\text { (9) }\end{array}$ & $\begin{array}{l}\text { Co-57, (122) } \\
\text { Co-57, (136) } \\
\text { Co-58, (811) } \\
\mathrm{Ag}-110 \mathrm{~m},(658) \\
\mathrm{Ag}-110 \mathrm{~m},(885) \\
\mathrm{Ag}-110 \mathrm{~m},(937) \\
\mathrm{Ag}-110 \mathrm{~m},(1384) \\
\mathrm{Mn}-54,(835) \\
\text { Co-60, }(1173) \\
\text { Co-60, (1332) } \\
\mathrm{K}-40,(1461)\end{array}$ & $\begin{array}{l}12551 \\
2186 \\
5144 \\
504 \\
349 \\
159 \\
78 \\
9724 \\
1944 \\
1842 \\
18.7\end{array}$ \\
\hline
\end{tabular}


activities were ${ }^{54} \mathrm{Mn}(834.8 \mathrm{keV}),{ }^{60} \mathrm{Co}(1173.2 \mathrm{keV}$, $1332.5 \mathrm{keV}),{ }^{40} \mathrm{~K}(1460.8 \mathrm{keV}),{ }^{57} \mathrm{Co}(122.1 \mathrm{keV})$ and ${ }^{58} \mathrm{Co}(810.8 \mathrm{keV})$, and ${ }^{124} \mathrm{Sb}(1690.0 \mathrm{keV})$, and in addition, ${ }^{110 \mathrm{~m}} \mathrm{Ag}$ (657.8 keV, $\left.884.7 \mathrm{keV}, 937.5 \mathrm{keV}, 1384.3 \mathrm{keV}\right)$. The ${ }^{110 \mathrm{~m}} \mathrm{Ag}$ (249.9d) activity is attributed to the
${ }^{109} \mathrm{Ag}(\mathrm{n}, \gamma){ }^{110 \mathrm{~m}} \mathrm{Ag}$ reaction from extensive Ag electrical contact coatings on antenna parts. Table 1 summarizes the gamma-ray energy spectroscopy measurements discussed above. All measurements were made with the same detector. Listed are the observed isotopes at each location

Table 2. Summary of activity measurements on samples removed from TFTR and counted about $390 \mathrm{~d}$ after shutdown. Shown are the sample description, mass (g), approximate major radius $\mathrm{R}(\mathrm{m})$, shortest distance from sample to Vessel Top (VT) or shortest distance from sample to Vessel Midplane (VM), the toroidal angle $\phi$ (degree), dominant activities, and activation per unit mass $(\mu \mathrm{Ci} / \mathrm{g}$ ).

\begin{tabular}{|c|c|c|c|c|c|c|c|c|c|c|c|c|c|}
\hline $\begin{array}{l}\text { Sample } \\
\text { Description }\end{array}$ & $\begin{array}{l}\text { Mass } \\
(\mathrm{g})\end{array}$ & $\mathrm{R}(\mathrm{m})$ & $\mathrm{d}(\mathrm{m})$ & $\phi$ & Isotop & $\begin{array}{l}\text { Activity } \\
\mu \mathrm{Ci} / \mathrm{g}\end{array}$ & $\begin{array}{l}\text { Sample } \\
\text { Description }\end{array}$ & $\begin{array}{l}\text { Mass } \\
(\mathrm{g})\end{array}$ & $\mathrm{R}(\mathrm{m})$ & $\mathrm{d}(\mathrm{m})$ & $\phi$ & Isotope & $\begin{array}{l}\text { Activity } \\
\mu \mathrm{Ci} / \mathrm{g}\end{array}$ \\
\hline $\begin{array}{l}\# 980061 \\
\text { SS bolt } \\
\text { from } \\
\text { glow probe }\end{array}$ & \begin{tabular}{|l|}
57.56 \\
\end{tabular} & 3.9 & $\begin{array}{l}2.13 \\
\text { from } \\
\text { VT }\end{array}$ & 270 & $\begin{array}{l}\text { Mn-54 } \\
\text { Co-57 } \\
\text { Co-58 } \\
\text { Co-60 }\end{array}$ & \begin{tabular}{l|}
$4.25 \mathrm{E}-5$ \\
$1.84 \mathrm{E}-5$ \\
$3.38 \mathrm{E}-6$ \\
$2.39 \mathrm{E}-4$
\end{tabular} & $\begin{array}{l}\text { \#980092 } \\
\text { brass bus } \\
\text { joint bolt }\end{array}$ & 175 & 3.9 & $\begin{array}{l}0.61 \\
\text { from } \\
\mathrm{VT}\end{array}$ & 180 & $\begin{array}{l}\mathrm{Mn}-54 \\
\mathrm{Co}-60\end{array}$ & \begin{tabular}{|l|}
$1.29 \mathrm{E}-5$ \\
$5.94 \mathrm{E}-4$
\end{tabular} \\
\hline $\begin{array}{l}\# 980062 \\
\text { brass bus } \\
\text { joint bolt }\end{array}$ & \begin{tabular}{|l}
174.00 \\
\end{tabular} & 3.9 & $\begin{array}{l}0.61 \\
\text { from } \\
\text { VT }\end{array}$ & 234 & $\begin{array}{l}\mathrm{Mn}-54 \\
\mathrm{Co}-60\end{array}$ & $\begin{array}{l}2.20 \mathrm{E}-5 \\
8.59 \mathrm{E}-4\end{array}$ & $\begin{array}{l}\text { \#980093 } \\
\text { SS bus } \\
\text { joint washer }\end{array}$ & 21.9 & 3.9 & $\begin{array}{l}0.61 \\
\text { from } \\
\text { VT }\end{array}$ & 180 & $\begin{array}{l}\text { Mn-54 } \\
\text { Co-57 } \\
\text { Co-58 } \\
\text { Co-60 }\end{array}$ & $\begin{array}{l}3.45 \mathrm{E}-4 \\
7.74 \mathrm{E}-3 \\
7.02 \mathrm{E}-4 \\
5.78 \mathrm{E}-3\end{array}$ \\
\hline $\begin{array}{l}\text { \#980063 Cu } \\
\text { /SS/plastic } \\
\text { ground strap }\end{array}$ & \begin{tabular}{|l|}
12.4 \\
\end{tabular} & 3.9 & $\begin{array}{l}0.20 \\
\text { from } \\
\text { VT }\end{array}$ & 126 & $\begin{array}{l}\text { Co-60 } \\
\text { Zn-65 } \\
\text { Sb-124 }\end{array}$ & $\begin{array}{l}3.04 \mathrm{E}-3 \\
1.45 \mathrm{E}-4 \\
7.68 \mathrm{E}-5\end{array}$ & $\begin{array}{l}\# 90094 \\
\text { Cu tritium } \\
\text { ground strap }\end{array}$ & 69.37 & 3.9 & $\begin{array}{l}1.82 \\
\text { from } \\
\text { VT }\end{array}$ & 9 & $\begin{array}{l}\text { Co-60 } \\
\text { Sb-124 }\end{array}$ & $\begin{array}{l}8.89 \mathrm{E}-5 \\
2.30 \mathrm{E}-6\end{array}$ \\
\hline $\begin{array}{l}\text { \#980064 } \\
\text { SS } \\
\text { support bolt }\end{array}$ & 7.39 & 3.9 & $\begin{array}{l}0.10 \\
\text { from } \\
\text { VT }\end{array}$ & 126 & $\begin{array}{l}\text { Mn-54 } \\
\text { Co-57 } \\
\text { Co-58 } \\
\text { Co-60 } \\
\end{array}$ & $\begin{array}{l}3.39 \mathrm{E}-3 \\
3.14 \mathrm{E}-3 \\
3.61 \mathrm{E}-4 \\
5.24 \mathrm{E}-3\end{array}$ & $\begin{array}{l}980095 \\
\text { Cu ground } \\
\text { strap }\end{array}$ & 66.41 & 3.9 & $\begin{array}{l}1.82 \\
\text { from } \\
\mathrm{VT}\end{array}$ & 63 & $\begin{array}{l}\text { Co-60 } \\
\text { Sb-124 }\end{array}$ & $\begin{array}{l}9.35 \mathrm{E}-5 \\
2.17 \mathrm{E}-6\end{array}$ \\
\hline $\begin{array}{l}\# 980071 \\
\text { brass bus } \\
\text { joint bolt }\end{array}$ & \begin{tabular}{|l|}
174.9 \\
\end{tabular} & 3.9 & $\begin{array}{l}0.46 \\
\text { from } \\
\text { VT }\end{array}$ & 0 & $\begin{array}{l}\text { Mn-54 } \\
\text { Co-60 }\end{array}$ & $\begin{array}{l}1.11 \mathrm{E}-4 \\
5.09 \mathrm{E}-4\end{array}$ & $\begin{array}{l}\# 980100 \\
\text { brass screws }\end{array}$ & 3.11 & 3.9 & $\begin{array}{l}0.10 \\
\text { from } \\
\text { VT }\end{array}$ & 126 & $\begin{array}{l}\text { Co-60 } \\
\text { Zn-65 }\end{array}$ & $\begin{array}{l}2.08 \mathrm{E}-3 \\
7.93 \mathrm{E}-3\end{array}$ \\
\hline $\begin{array}{l}\text { \#980072 } \\
\text { SS bus } \\
\text { joint } \\
\text { washer }\end{array}$ & 21.99 & 3.9 & $\begin{array}{l}0.46 \\
\text { from } \\
\text { VT }\end{array}$ & $\overline{0}$ & $\begin{array}{l}\text { Mn-54 } \\
\text { Co-57 } \\
\text { Co-58 } \\
\text { Co-60 } \\
\end{array}$ & $\begin{array}{l}3.04 \mathrm{E}-4 \\
6.91 \mathrm{E}-3 \\
6.48 \mathrm{E}-4 \\
5.47 \mathrm{E}-3\end{array}$ & $\begin{array}{l}980101 \\
\text { SS } \\
\text { support nut }\end{array}$ & 3.11 & 3.9 & $\begin{array}{l}0.10 \\
\text { from } \\
\text { VT }\end{array}$ & 126 & $\begin{array}{l}\mathrm{Mn}-54 \\
\text { Co-57 } \\
\text { Co-58 } \\
\text { Co-60 } \\
\end{array}$ & $\begin{array}{l}4.58 \mathrm{E}-3 \\
3.27 \mathrm{E}-3 \\
3.57 \mathrm{E}-4 \\
4.85 \mathrm{E}-3\end{array}$ \\
\hline $\begin{array}{l}\# 980073 \\
\text { brass/plastic } \\
\text { Swagelock } \\
\text { fitting }\end{array}$ & 12.63 & 4.7 & $\begin{array}{l}0.91 \\
\text { from } \\
V M\end{array}$ & 0 & $\begin{array}{l}\text { Co-60 } \\
\text { Zn-65 }\end{array}$ & $\begin{array}{l}2.12 \mathrm{E}-4 \\
9.63 \mathrm{E}-4\end{array}$ & $\begin{array}{l}\# 980102 \\
\text { brass bus } \\
\text { joint bolt }\end{array}$ & 175 & 3.9 & $\begin{array}{l}0.46 \\
\text { from } \\
\text { VT }\end{array}$ & 126 & $\begin{array}{l}\text { Mn-54 } \\
\text { Co-60 }\end{array}$ & $\begin{array}{l}1.01 \mathrm{E}-4 \\
4.76 \mathrm{E}-4\end{array}$ \\
\hline $\begin{array}{l}\text { \#980074 } \\
\text { SS/plastic } \\
\text { Swagelock } \\
\text { fitting }\end{array}$ & 11.88 & 4.6 & $\begin{array}{l}0.76 \\
\text { from } \\
V M\end{array}$ & 0 & $\begin{array}{l}\text { Mn-54 } \\
\text { Co-57 } \\
\text { Co-58 } \\
\text { Co-60 }\end{array}$ & $\begin{array}{l}1.14 \mathrm{E}-3 \\
8.13 \mathrm{E}-4 \\
1.09 \mathrm{E}-4 \\
2.15 \mathrm{E}-3\end{array}$ & $\begin{array}{l}\# 98103 \\
\text { SS } \\
\text { bus joint } \\
\text { washer }\end{array}$ & 21.9 & 3.9 & $\begin{array}{l}0.46 \\
\text { from } \\
\text { VT }\end{array}$ & 126 & $\begin{array}{l}\text { Mn-54 } \\
\text { Co-57 } \\
\text { Co-58 } \\
\text { Co-60 }\end{array}$ & $\begin{array}{l}2.79 \mathrm{E}-4 \\
5.70 \mathrm{E}-3 \\
6.14 \mathrm{E}-4 \\
6.24 \mathrm{E}-3\end{array}$ \\
\hline $\begin{array}{l}\# 980075 \\
\text { support } \\
\text { plate }\end{array}$ & 163.5 & 5.32 & $\begin{array}{l}1.52 \\
\text { from } \\
\text { VM }\end{array}$ & 0 & Co-60 & $2.50 \mathrm{E}-5$ & $\begin{array}{l}\text { \#980104 } \\
\text { brass } \\
\text { bus joint bolt }\end{array}$ & 175 & 3.9 & $\begin{array}{l}1.52 \\
\text { from } \\
\text { VT }\end{array}$ & 288 & $\begin{array}{l}\text { Mn-54 } \\
\text { Co-60 }\end{array}$ & $\begin{array}{l}1.10 \mathrm{E}-4 \\
6.11 \mathrm{E}-4\end{array}$ \\
\hline $\begin{array}{l}\# 980076 \\
\text { Cu/plastic } \\
\text { ground strap }\end{array}$ & 3.9 & 3.9 & $\begin{array}{l}2.01 \\
\text { from } \\
V M\end{array}$ & 270 & Co-60 & $1.42 \mathrm{E}-4$ & $\begin{array}{l}\# 980105 \\
\text { SS } \\
\text { bus joint } \\
\text { washer }\end{array}$ & 22 & 3.9 & $\begin{array}{l}1.52 \\
\text { from } \\
\text { VT }\end{array}$ & 288 & $\begin{array}{l}\text { Mn-54 } \\
\text { Co-57 } \\
\text { Co-58 } \\
\text { Co-60 }\end{array}$ & $\begin{array}{l}3.65 \mathrm{E}-4 \\
8.19 \mathrm{E}-3 \\
7.40 \mathrm{E}-4 \\
6.19 \mathrm{E}-3\end{array}$ \\
\hline $\begin{array}{l}\text { \#980082 } \\
\text { brass bus } \\
\text { joint bolt }\end{array}$ & 128.6 & 3.9 & $\begin{array}{l}0.81 \\
\text { from } \\
\text { VT }\end{array}$ & 0 & $\begin{array}{l}\text { Mn-54 } \\
\text { Co-57 } \\
\text { Co-58 } \\
\text { Co-60 }\end{array}$ & $\begin{array}{l}1.83 \mathrm{E}-4 \\
9.07 \mathrm{E}-5 \\
1.76 \mathrm{E}-5 \\
2.13 \mathrm{E}-3\end{array}$ & $\begin{array}{l}\# 980124 \\
\text { SS } \\
\text { support bolt }\end{array}$ & 21.3 & 5.0 & $\begin{array}{l}1.22 \\
\text { from } \\
\mathrm{VM}\end{array}$ & $\overline{0}$ & $\begin{array}{l}\mathrm{Mn}-54 \\
\text { Co-57 } \\
\text { Co-58 } \\
\text { Co-60 }\end{array}$ & $\begin{array}{l}3.45 \mathrm{E}-4 \\
2.59 \mathrm{E}-4 \\
3.37 \mathrm{E}-5 \\
8.81 \mathrm{E}-4\end{array}$ \\
\hline $\begin{array}{l}980083 \\
\text { SS bus joint } \\
\text { washer }\end{array}$ & 31.26 & 3.9 & $\begin{array}{l}0.81 \\
\text { from } \\
\mathrm{VT}\end{array}$ & 0 & $\begin{array}{l}\text { Mn-54 } \\
\text { Co-57 } \\
\text { Co-58 } \\
\text { Co-60 }\end{array}$ & $\begin{array}{l}3.57 \mathrm{E}-4 \\
2.06 \mathrm{E}-4 \\
2.04 \mathrm{E}-5 \\
1.12 \mathrm{E}-3\end{array}$ & $\begin{array}{l}\text { \#980126 } \\
\text { SS insulator } \\
\text { bolt }\end{array}$ & 32.17 & 5.0 & $\begin{array}{l}1.22 \\
\text { from } \\
\mathrm{VM}\end{array}$ & 72 & $\begin{array}{l}\text { Mn-54 } \\
\text { Co-57 } \\
\text { Co-58 } \\
\text { Co-60 }\end{array}$ & $\begin{array}{l}2.17 \mathrm{E}-4 \\
1.41 \mathrm{E}-4 \\
1.72 \mathrm{E}-5 \\
4.44 \mathrm{E}-4\end{array}$ \\
\hline $\begin{array}{l}\text { \#980090 } \\
\text { SS support } \\
\text { bracket }\end{array}$ & 42.1 & 3.9 & $\begin{array}{l}0.46 \\
\text { from } \\
\text { VT }\end{array}$ & 126 & $\begin{array}{l}\text { Mn-54 } \\
\text { Co-57 } \\
\text { Co-58 } \\
\text { Co-60 }\end{array}$ & $\begin{array}{l}2.37 \mathrm{E}-3 \\
1.40 \mathrm{E}-3 \\
1.52 \mathrm{E}-4 \\
2.80 \mathrm{E}-3\end{array}$ & $\begin{array}{l}\text { \#980127 } \\
\text { SS insulator } \\
\text { washer }\end{array}$ & 7 & 5.0 & $\begin{array}{l}1.22 \\
\text { from } \\
V M\end{array}$ & 72 & $\begin{array}{l}\text { Mn-54 } \\
\text { Co-57 } \\
\text { Co-58 } \\
\text { Co-60 }\end{array}$ & $\begin{array}{l}3.54 \mathrm{E}-4 \\
4.06 \mathrm{E}-4 \\
2.63 \mathrm{E}-5 \\
1.31 \mathrm{E}-3\end{array}$ \\
\hline $\begin{array}{l}\# 980091 \\
\text { SS support } \\
\text { bolt }\end{array}$ & 18.77 & 3.9 & $\begin{array}{l}0.46 \\
\text { from } \\
\text { VT }\end{array}$ & 126 & $\begin{array}{l}\text { Mn-54 } \\
\text { Co-57 } \\
\text { Co-58 } \\
\text { Co-60 }\end{array}$ & $\begin{array}{l}2.05 \mathrm{E}-3 \\
2.04 \mathrm{E}-3 \\
2.33 \mathrm{E}-4 \\
4.34 \mathrm{E}-3\end{array}$ & & & & & & & \\
\hline
\end{tabular}


and the measured detector counts per minute uncorrected for detector absolute efficiency. There are a number of difficult issues involved in attempting to convert the measured detector count rate for a particular isotopic gamma-ray energy to yield the absolute activity of the source in the TFTR Test Cell. In particular, these are extended sources of gamma-rays with varying geometries, gamma-ray absorption, attenuation, shielding, scattering, and angle of incidence on the detector. The available absolute efficiency calibration procedures usually involve small point or disk calibration sources on a known backing, placed in front of a detector to give an accurately defined solid angle with minimal scattering and background effects. The complexity of the TFTR experimental configuration prevents this approach in a manner that would yield sufficient accuracy for benchmarking purposes.

In view of these difficulties, an alternate approach was pursued to obtain an activity calibration of the global TFTR activation decay with sufficient accuracy to provide benchmarking for the present measurements and future simulations. Small activated TFTR parts, fabricated from representative TFTR materials were removed from well characterized vessel and near-vessel locations. The activations of these samples were measured in the PPPL Radiological and Environmental Measurements Laboratory about 390 d after shutdown using high resolution, high efficiency, low background, gamma-ray spectroscopy systems with efficiency calibrations traceable to NIST standards. The results of these measurements are summarized in Table 2. Shown are the sample description, exposure location, mass, dominant activities and activation per unit mass. Future work will use these and the results of similar measurements in progress to benchmark simulations of the TFTR cool-down for testing low activation reactor design codes

\section{SUMMARY AND CONCLUSIONS}

The measurements have characterized residual TFTR neutron activation. The results indicate that, in general, the observed gamma activities are similar to those expected from previous simulation work, and can be used for several applications, including accurate determinations of low activation long-lived cooling, and benchmarking advanced simulations of D-T fusion reactor neutron activation.

\section{ACKNOWLEDGMENTS}

The technical contributions of Keith Chase and Joseph Winston are gratefully acknowledged. This work was supported by US DOE Contract No. DE-AC02$76 \mathrm{CH} 03073$.

\section{REFERENCES}

1. R. J. Hawryluk, et al., "Fusion Plasma Experiments on TFTR: A 20 Year Retrospective", Phys. Plasmas, 5(5) 1577 (1998).

2. L.-P. $\mathrm{Ku}$ and J. G. Kolibal, "Radioactivation Characteristics for the Tokamak Fusion Test Reactor", Nuc. Technol./Fus.,4 (11), 586 (1983).

3. L. P. Ku and S. L. Liew, "Global Dose Rate in TFTR Due to Neutron Induced Residual Radioactivities During DT Operation", Procedings of the 15th IEEE/NPSS Symposium on Fusion Engineering, p 313, October 1115, 1993, Hyannis, MA, and references therein.

4. H. W. Kugel et al., "Measurements of TFTR D-T Radiation Shielding Efficiency", Fus. Eng. \& Design, 28, 534 (1995). 